\title{
Investigation of long waviness induced by the wire saw process
}

\author{
E Teomete \\ Izmir Institute of Technology, Gulbahce Kova, Urla, Izmir, Turkey. email: eteomete@gmail.com
}

The manuscript was received on 4 May 2010 and was accepted after revision for publication on 15 September 2010.

DOI: 10.1177/2041297510393620

\begin{abstract}
In the photovoltaic and semiconductor industries silicon wafers are sliced using the wire saw process. This process is also used to machine almost all kinds of brittle materials where high yield and low surface damage is desired. The wire saw process induces roughness and long waviness on the cut surface. These defects have to be removed by post-process techniques including grinding and lapping, which increases costs. The present study investigated the long waviness induced by the wire saw process. An analytical model for long waviness generation has been developed. Experimental work was conducted with different process parameters. The analytical model is capable of explaining the long waviness generation observed in experimental work. Process design recommendations with minimal waviness and high efficiency are presented.
\end{abstract}

Keywords: wire saw, surface waviness, ceramic, model

\section{INTRODUCTION}

The wire saw process is widely used in slicing silicon wafers in the photovoltaic and microelectronics industries. The advantages of the wire saw with respect to inner diameter saw are high productivity, less wafer surface damage, and lower kerf loss [1]. The wire saw process can also be used to cut all kinds of brittle materials including sapphire, silicon carbide, lithium niobate, rocks, high-density ceramics, and foam ceramics $[\mathbf{1}-\mathbf{3}]$.

In the 1990s, the wire saw process consisted of a bare steel wire and abrasive-carrying slurry, resulting in free-abrasive machining using elasto-hydrodynamic forces $[\mathbf{4}, \mathbf{5}]$. In order to increase the efficiency and to cut harder materials, the diamond-impregnated wire saw process, which leads to fixed-abrasive machining, was developed [4]. The diamond-impregnated wire saw process also eliminated the disposal of high amounts of hazardous slurry, which was an output of the abrasive-carrying slurry wire saw process [6].
The wire saw process makes up 30 per cent of the total silicon wafer production cost, which affects the industry directly. The high cost of the process requires the development of models relating process parameters with outputs [7]. There have been three main topics in wire saw literature: material removal mechanisms, kinematics of the wire, and parametric studies between the process inputs and outputs.

The material removal rate of the abrasive-carrying slurry wire saw process, which leads to free-abrasive machining, has been studied by different researchers $[5,7]$. The material removal mechanism for beadimpregnated wire saw cutting of rock is a Hertziantype fracture that occurs due to the tensile field resulting from the sliding bead [8]. Wire vibration characteristics have been studied with respect to process parameters $[\mathbf{1}, \mathbf{9}, \mathbf{1 0}]$. The influence of process parameters on slurry film pressure and thickness has been studied for the free-abrasive wire saw process $[\mathbf{1}, \mathbf{5}]$. Experimental parametric studies relating surface damage with process parameters for cutting various materials have also been conducted $[2,6,11]$. 
Rigorous efforts have been devoted to removing wire saw-induced waviness by post-processing techniques [12-17].

In the present study, the long waviness generated by the wire saw process was investigated. An experimental study was conducted to explore the effects of process parameters. An analytical model which is based on the work of Bastawros et al. [18] was compared with the experimental observations.

\section{EXPERIMENTAL DESIGN}

Wire saw experiments were conducted on alumina ceramic. The wire bow angle $\alpha$, wire axial speed $V_{x}$, and feed rate $V_{z}$ were measured during the wire saw cutting tests. The surface waviness of cut surfaces was also measured. The equipment used in these measurements and the process parameters are presented in this section.

\subsection{The wire saw process and wire bow angle measurement}

A wire saw machine (Millennium model; Diamond Wire Technology, Colorado Springs, Colorado, USA) was used in the experiments. This spool-to-spool wire saw machine with rocking motion of the wire can be controlled by the wire speed $V_{x}$, down-feed speed $V_{z}$, and wire tension $T$. The tension is controlled by wire tension pulleys powered by air pressure, while the rocking motion is controlled by wire guide pulleys, as can be seen in Fig. 1. The cut length of wire, which is used during the cutting, is $310 \mathrm{ft}(94.5 \mathrm{~m})$. Thus, at every direction reversal, $310 \mathrm{ft}$ of wire is transferred from one spool to the other.

The wire speed is a function of spool diameter as the machine controls the speed via the angular frequency of the spool. In order to get the actual average speeds, a Strobex Systems model 236 stroboscope (Chadwick-Helmuth Inc., El Monte, California, USA) was used. The down-feed speed $V_{z}$ was measured by tracking the position of a grid, which is on the yoke of the machine, with respect to a stationary point on the frame of the machine. As the yoke goes down, the grid moves relative to the fixed point on the frame of the machine. The time to travel $5 \mathrm{~mm}$ grids was measured during the tests.

Diamond-grit-coated steel wire (a product of Well Diamond Wire Saws Inc., Norcross, Georgia, USA) was used in the experiments. The wire is manufactured by mechanically impregnating the diamond grits into the steel wire. The impregnation process might be successful for some grits while others just leave an indentation mark on the wire and cannot be affixed, as seen in Fig. 2.
Coolant consisting of a mixture of water and lubricant (Sawzit; Synthetic Lubricants, Inc., Greenville, Michigan, USA) at a ratio of 50:1 was used during the cutting tests.

Alumina ceramic samples having tensile strength $\sigma_{\text {fr }}=300 \mathrm{MPa}$, Poisson's ratio $\nu=0.22$, fracture toughness $K_{\mathrm{IC}}=4 \mathrm{MPa} \mathrm{m}{ }^{0.5}$, Young's modulus $E=370 \mathrm{GPa}$ [19], and hardness $H=22 \mathrm{GPa}$ [20] were used in the

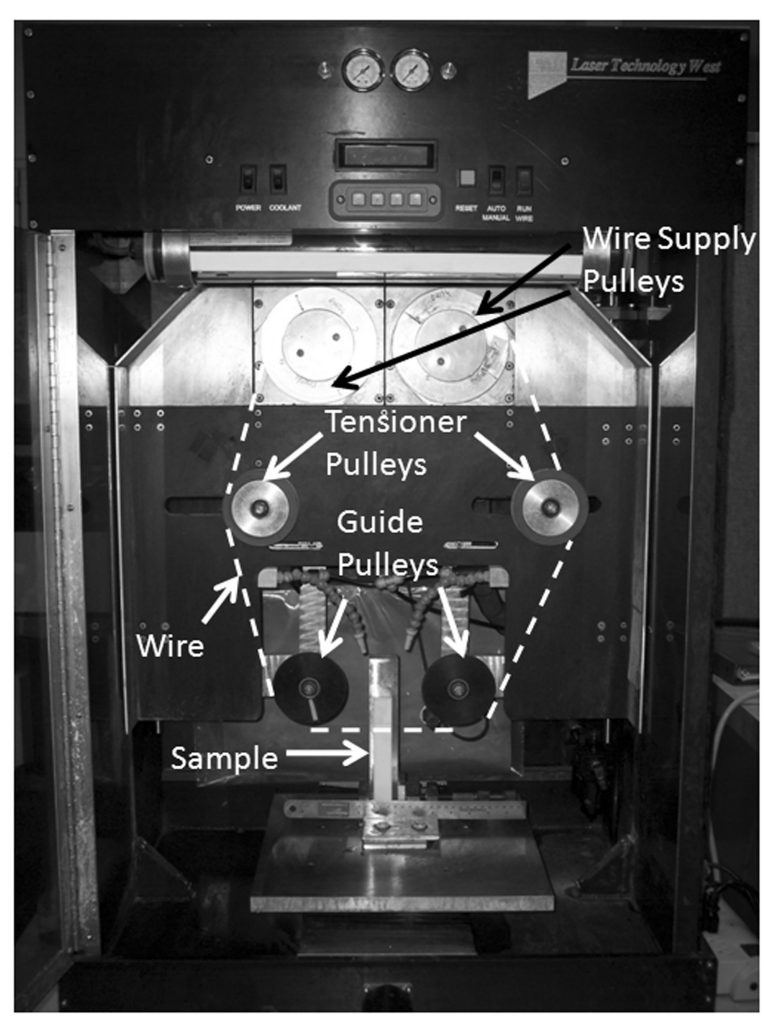

Fig. 1 Single-wire, spool-to-spool wire saw machine (DWT Inc., Millennium model) used in waviness tests. The wire track is marked by the dashed line

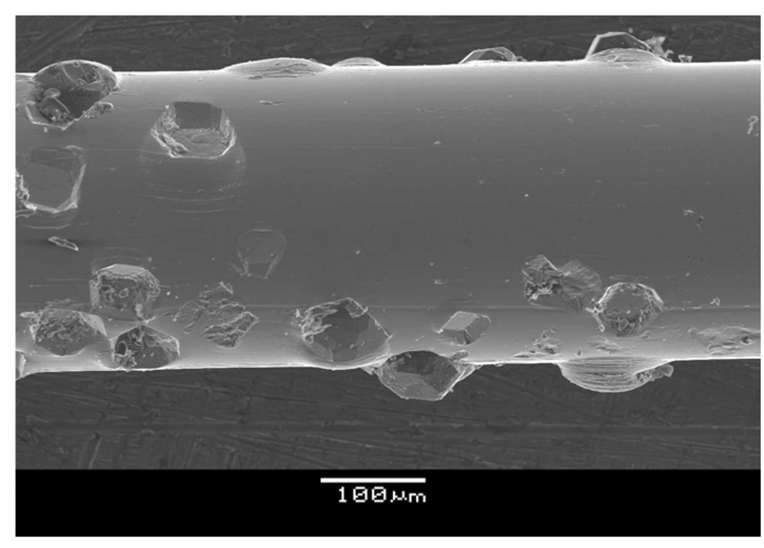

Fig. 2 Scanning electron micrograph of diamondimpregnated wire 
Table 1 Process parameter values used in the long waviness tests

\begin{tabular}{lllll}
\hline & $V_{x}(\mathrm{~m} / \mathrm{s})$ & $V_{z}(\mu \mathrm{m} / \mathrm{s})$ & $T(\mathrm{~N})$ & $L_{\mathrm{o}}(\mathrm{mm})$ \\
\hline TC20 & 2 & 6.35 & 13.3 & 25 \\
TC21 & 2 & 6.35 & 26.7 & 25 \\
TC22 & 2 & 12.7 & 26.7 & 25 \\
\hline
\end{tabular}

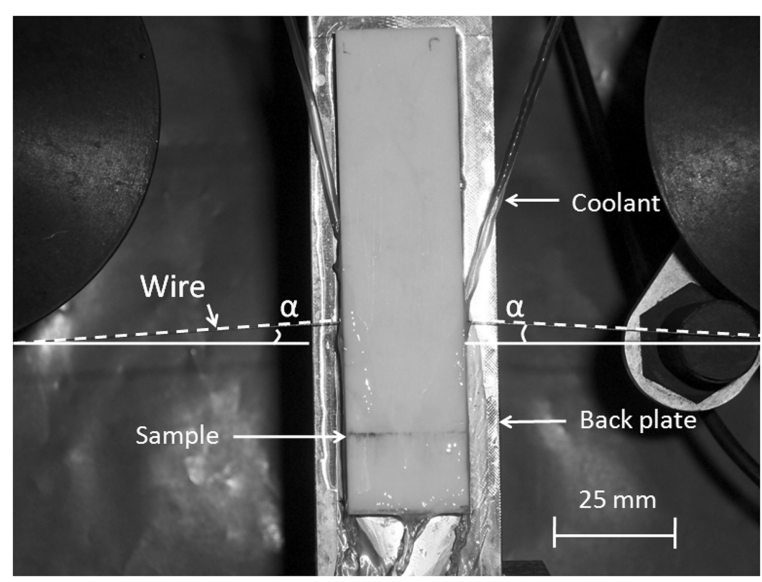

Fig. 3 Optical image showing the wire inclination angle with the cutting direction at the entrance and exit from the specimen for a waviness test

cutting tests. The cut length of the samples is $L_{\mathrm{o}}=25 \mathrm{~mm}$ and the height is $H_{\mathrm{s}}=60-90 \mathrm{~mm}$. Three tests were done with the process parameters presented in Table 1.

A Kodak Easy Share DX 76306.1 megapixel digital camera was used to measure the wire bow angle as seen in Fig. 3. The images of the wire and sample were collected during the test and analysed using the MATLAB image-processing toolbox to obtain the angle $\alpha$ between the wire and the horizontal. The average of the steady-state wire bow angles $\alpha$ attained in the test was taken as the steady-state wire bow angle of that test.

The Kodak digital camera was also used to obtain the profile of the wire marks on the cut surface as seen in Fig. 4. The cut surface images were processed using the image-processing toolbox of MATLAB to obtain the wire mark profile for each test.

\subsection{Surface waviness measurements}

The surface waviness of the cut surfaces was measured using an optical non-contact profilometer (Zygo New View 6000; Zygo Corporation, Middlefield, Connecticut, USA). A $2.5 \times$ lens with $2 \times$ zoom was used for the measurements. The profilometer has a vertical resolution of the order of $3 \mathrm{~nm}$;

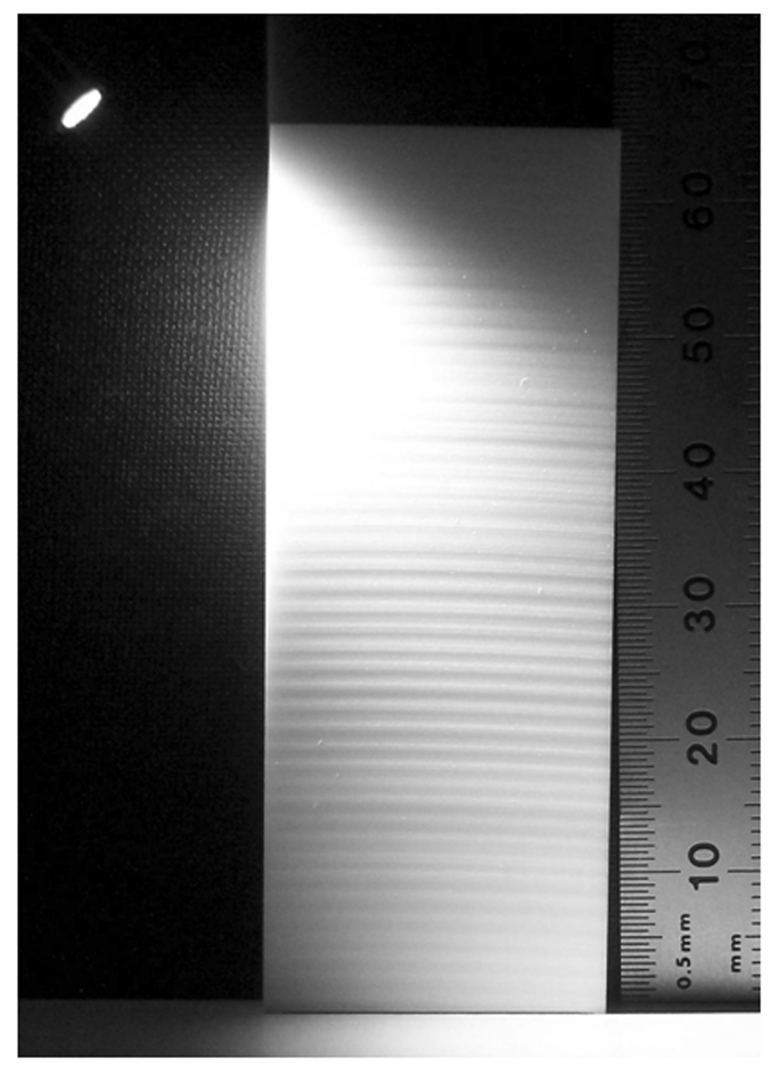

Fig. 4 Wire marks on a wire saw-cut surface (TC22; $V_{x}=2 \mathrm{~m} / \mathrm{s}, V_{z}=12.7 \mu \mathrm{m} / \mathrm{s}, T=26.7 \mathrm{~N}$ )

the resolution in the horizontal plane is $8.83 \mu \mathrm{m}$, while the field of view used is $1.41 \mathrm{~mm} \times 1.06 \mathrm{~mm}$.

In a stitch measurement, the profilometer took continuous measurements of $1.41 \mathrm{~mm} \times 1.06 \mathrm{~mm}$ and stitched them together into one dataset. Stitch measurements were applied in the direction of cutting at the centre of the cut surface for each sample. After the measurements were taken, the data were processed using the software Metro Pro version 8.1.5 developed by Zygo Corporation. The best-fit plane was removed and a low-pass filter was applied to obtain the waviness profile of the cut surface.

\section{WIRE SAW PROCESS-INDUCED WAVINESS MODEL}

The wire bows in the plane of the cut ( $X-Z$ plane) and in the orthogonal $(X-Y)$ plane. The definitions of the planes are presented in Fig. 5. The string deflection model is used to explain the wire bow in the $X-Z$ plane, while energy competition between the oblique cutting forces, the free-standing wire, and wire tension is used to explain the wire deflection in the $X-Y$ plane. The models are based on the work of Bastawros et al. [18]. 


\subsection{The string deflection model}

The deformation of a wire that is tensioned at both ends and subject to a distributed load per unit length, $w$, in the middle is presented in Fig. 6. An infinitesimal region of the loaded string is presented in Fig. 7. The horizontal and vertical equilibrium equations for force are obtained using Fig. 7 and presented in equations (1) and (2), respectively

$$
\begin{aligned}
& T_{1} \cos \theta_{1}=T_{2} \cos \theta_{2}=T \\
& T_{2} \sin \theta_{2}+w(x) \Delta x=T_{1} \sin \theta_{1}
\end{aligned}
$$

Solving equation (1) for $T_{1}$ and $T_{2}$ and inserting into equation (2) yields

$$
T\left(\tan \theta_{2}-\tan \theta_{1}\right)=-w(x) \cdot \Delta x
$$

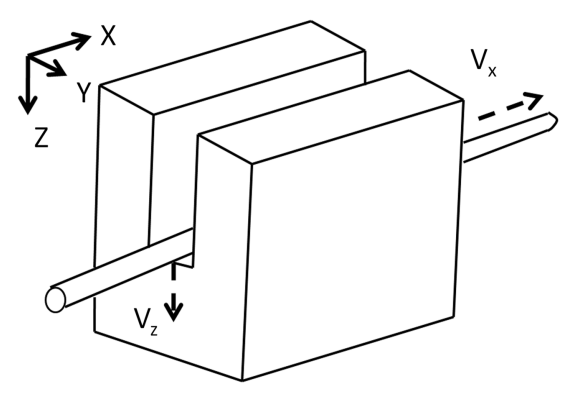

Fig. 5 The coordinate system defined for the wire saw cutting process

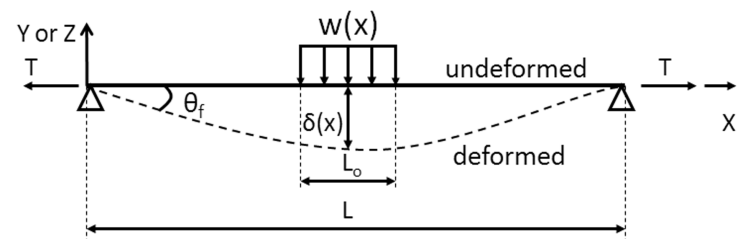

Fig. 6 The model of a wire under tension and distributed load

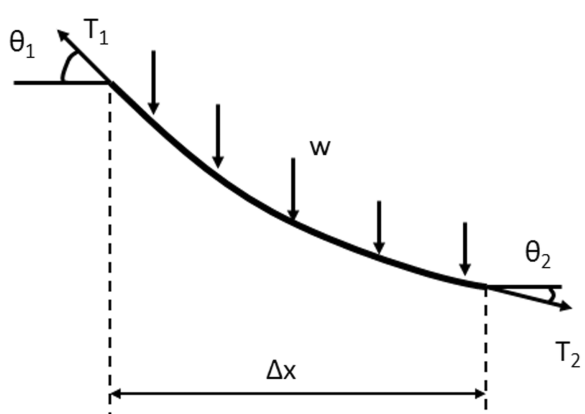

Fig. 7 The forces acting on an infinitesimal string element
The string deflection is $\delta(x)$ as seen in Fig. 6. The slope of the string at any point can be written as

$$
\tan \theta=\frac{\mathrm{d} \delta(x)}{\mathrm{d} x}
$$

Equation (3) can be updated using equation (4)

$$
T\left(\frac{\mathrm{d} \delta(x) /\left.\mathrm{d} x\right|_{2}-\mathrm{d} \delta(x) /\left.\mathrm{d} x\right|_{1}}{\Delta x}\right)=-w(x)
$$

In the limit where $\Delta x$ approaches zero, equation (5) can be written as

$$
T \frac{\mathrm{d}^{2} \delta(x)}{\mathrm{d} x^{2}}=-w(x)
$$

The distributed load per unit length of the wire is

$$
w(x)= \begin{cases}0, & 0 \leq x<\frac{L-L_{0}}{2} \\ w, & \frac{L-L_{0}}{2} \leq x \leq \frac{L+L_{0}}{2} 2 \\ 0, & \frac{L+L_{0}}{2}<x \leq L\end{cases}
$$

Using the boundary conditions $\delta(0)=0, \delta(L)=0$, and the continuity of slope and deflection of wire, equation (6) can be solved. The wire deflection is presented in equation (8). The displacement at the mid span of the wire is presented in equation (9). The maximum wire bow deflection in the $X-Z$ plane in the cut length seen in Fig. 6 is presented in equation (10). In these equations, $w$ is the distributed load, $L_{\mathrm{o}}$ is the cut length of the sample where the distributed load is applied, $T$ is the wire tension, and $L$ is the span of the wire between guide pulleys, as seen in Fig. 6

$$
\begin{aligned}
& \delta(x)=\frac{w L_{0}}{T} \\
& \times \begin{cases}\frac{1}{2} x, & 0 \leq x<\frac{L-L_{0}}{2} \\
-\frac{x^{2}}{2 L_{0}}+\frac{L x}{2 L_{0}}-\frac{1}{2 L_{0}}\left(\frac{L-L_{0}}{2}\right)^{2}, & \frac{L-L_{0}}{2} \leq x \leq \frac{L+L_{0}}{2} \\
\frac{1}{2}(L-x), & \frac{L+L_{0}}{2}<x \leq L\end{cases} \\
& \delta\left(\frac{L}{2}\right)=\frac{w L_{0}}{8 T}\left(2 L-L_{0}\right) \\
& \delta_{\max }=\delta\left(\frac{L}{2}\right)-\delta\left(\frac{L-L_{0}}{2}\right)=\frac{w L_{0}^{2}}{8 T}
\end{aligned}
$$

The wire deflection model presented in equation (8) is compared with the experimentally obtained wire bow in the $X-Z$ plane in section 4 . 


\subsection{The lateral deflection model in the $X-Y$ plane}

The string model presented in equations (8) to (10) is the upper bound for the wire deflection in the $X-Y$ plane. The wire deflection in the $X-Y$ plane is controlled by energy competition between the oblique cutting forces, the wire tension, and the freestanding wire loaded with a distributed load. Wire saw cutting occurs due to the cutting action of the individual grits in the cut length. Energetically, the cutting action of the individual grits is oblique cutting, which leads to divergence of the wire in the $X-Y$ plane and forms the long waviness. The total oblique load $w_{y}$ acting on the wire is related to the normal load $w_{z}$ with a proportionality factor $\beta$ as follows

$$
w_{y}=\beta \times w_{z}
$$

Experimental observations showed that the deflection in the $X-Y$ plane, $\delta_{y}$ increases up to a limit as the cut depth $z$ increases. As deflection in the $X-Y$ plane occurs due to oblique cutting action, the proportionality between deflection in the $X-Y$ plane, $\delta_{y}$, and the cut depth, $z$, is the same constant of proportionality as between lateral oblique force, $w_{y}$, and normal force, $w_{z}$

$$
\delta_{y}(L / 2, z)=\beta \times z
$$

The value of the proportionality factor, $\beta$, is determined from the surface profiles of each test using equation (12).

\subsection{The energy terms for surface waviness generation}

The long waviness occurs due to wire drifting in the $X-Y$ plane. The oblique cutting forces drift the wire out of the cutting plane $(X-Z)$, while the wire tension is applying a recovery work on the wire to bring the wire into the cutting plane $(X-Z)$. The limiting energy for the maximum deflection can be found using the work done by the free-standing wire under oblique load, $w_{y}$. Three energetic terms governing the behaviour of the wire are introduced in this section.

\subsubsection{Work done by the free-standing wire}

The upper bound of the work done by the free-standing wire, $W D_{\mathrm{fsw}}$, is calculated at maximum lateral deflection of the wire in the $X-Y$ plane, as in equation (13). The maximum deflection of the wire at mid span, $\delta_{y}^{*}$, in equation (9) is used to calculate the final result in equation (13)

$$
W D_{\mathrm{fsw}}=\int_{\left(L-L_{0}\right) / 2}^{\left(L+L_{0}\right) / 2} w_{y} \delta_{y}^{*}(x) \mathrm{d} x=\frac{w_{y}^{2} L_{\mathrm{o}}^{2}}{8 T}\left(2 L-L_{\mathrm{o}}\right)
$$

\subsubsection{Work done by the oblique cutting forces}

The work done by the oblique cutting force, $W D_{\text {obl }}$, drifts the wire in the $X-Y$ plane. The work done by the oblique cutting force is calculated using lateral distributed load, $w_{y}$, minus the lateral reaction, $R(z)$, applied by the wall of the sample to the wire. The lateral reaction on the wire $R(z)$ changes with depth of cut as seen in Fig. 8. The lateral reaction has its maximum value at the beginning of the cut and diminishes to zero at maximum lateral deflection $\delta_{y}^{*}$ obtained at the cut depth $z_{1}$. The lateral reaction is presented in equation (14). The work done due to oblique cutting is presented in equation (15). Using equations (12) and (15), equation (16) is obtained for the work done by oblique cutting

$$
\begin{aligned}
& R(z)=w_{y}\left(1-z / z_{1}\right) \\
& \begin{aligned}
W D_{\mathrm{obl}}(z) & =\int_{0}^{L_{0}}\left[w_{y}-R(z)\right] \delta_{y}(x, z) \mathrm{d} x \\
& =\left[w_{y}-R(z)\right] L_{0} \delta_{y}(L / 2, z)
\end{aligned} \\
& W D_{\mathrm{obl}}(z)=\beta L_{0} w_{y} z^{2} / z_{1}
\end{aligned}
$$

\subsubsection{Work done by the wire tension}

The work done by wire tension, $W D_{T}$, to deflect the wire back to the undeformed position in the $X-Y$ plane is

$$
\begin{aligned}
W D_{\mathrm{T}} & =\int_{0}^{\theta_{\mathrm{f}}} 2 T \sin \theta \frac{L}{2} d \theta \\
& =T L \int_{0}^{\theta_{\mathrm{f}}} \sin \theta \mathrm{d} \theta=T L\left(1-\cos \theta_{\mathrm{f}}\right)
\end{aligned}
$$

The angle $\theta_{\mathrm{f}}$ is between the wire and the horizontal line in the $X-Y$ plane as seen in Fig. 6. $\theta_{\mathrm{f}}$ is a function of wire length and mid-span deflection as presented in equation (18). The work done by wire tension can be

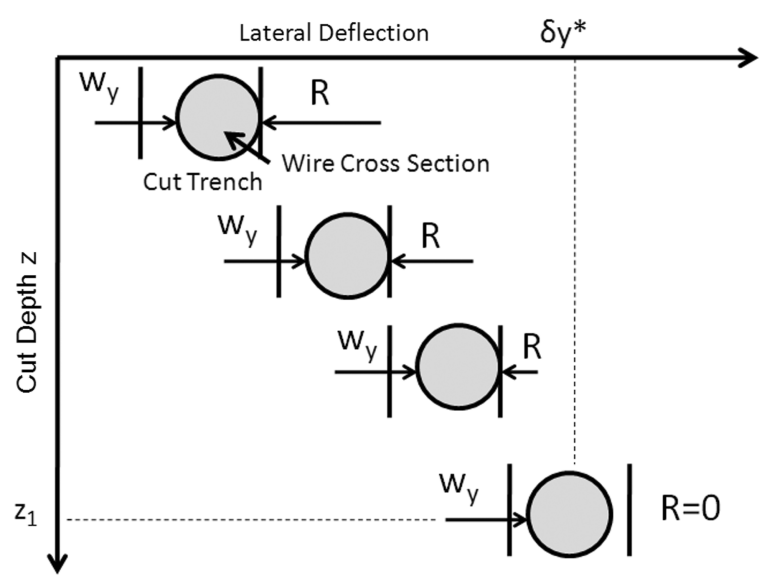

Fig. 8 The variation of lateral reaction with cut depth 
written using equations (12), (17), and (18), as presented in equation (19)

$$
\begin{aligned}
& \cos \theta_{\mathrm{f}}=\sqrt{\frac{L^{2}}{L^{2}+4 \delta_{y}^{2}(L / 2, z)}} \\
& W D_{\mathrm{T}}=T L\left(1-\frac{L}{\sqrt{L^{2}+4 \beta^{2} z^{2}}}\right)
\end{aligned}
$$

\subsection{The mechanisms affecting long waviness}

The mechanisms affecting the long waviness formation via the energetic terms presented in section 3.3 are examined in this section.

\subsubsection{Mechanism 1}

Mechanism 1 occurs when the oblique cutting forces drifts the wire in the $X-Y$ plane until the critical cut depth $z_{1}$ is achieved. The work done by oblique cutting $W D_{\text {obl }}$ increases with depth of cut until the critical cut depth $z_{1}$, at which $W D_{\text {obl }}$ is equal to the work done by the free-standing wire $W D_{\mathrm{fsw}}$, as presented in equation (20) and Fig. 9. At cut depths greater than $z_{1}$, the wire will continue with the same lateral deflection until a process disturbance changes the oblique cutting conditions and drifts the wire back into the cutting plane $X-Z$, as seen in Fig. 10. The rate of work done by oblique cutting is greater than the rate of work done by wire tension, as presented in equation (21). Mechanism 1 occurs when the wire tension is low, which leads to a flexible wire. Mechanism 1 leads to tapered edges and high-frequency roughness in the middle of the cut surface as seen in Fig. 10

$$
\begin{aligned}
& W D_{\mathrm{obl}}=W D_{\mathrm{fsw}} \\
& \frac{\partial W D_{\mathrm{obl}}}{\partial z}>\frac{\partial W D_{\mathrm{T}}}{\partial z}
\end{aligned}
$$

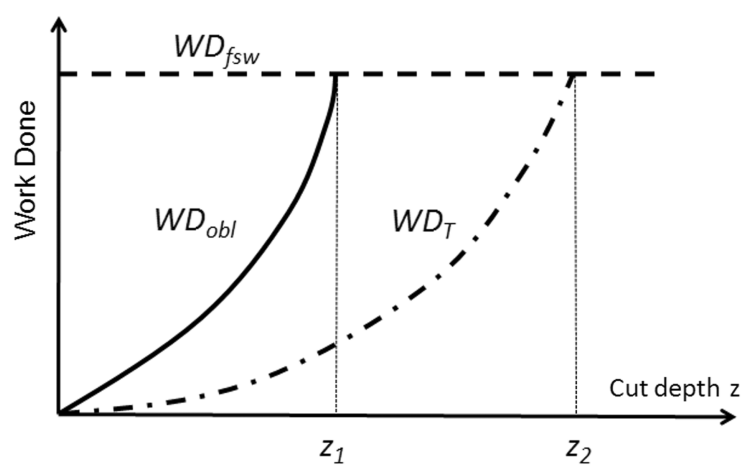

Fig. 9 Energy plot for mechanism 1

\subsubsection{Mechanism 2}

Mechanism 2 occurs when the oblique cutting forces drift the wire in the $X-Y$ plane until reaching the cut depth $z_{3}$, at which the work done by oblique cutting is equal to the work done by wire tension as seen in equation (22) and Fig. 11. Work done by wire tension will reverse the oblique cutting direction, drifting the wire back into the cutting plane $X-Z$ and forming waviness as seen in Fig. 12. The repetition of this process will lead to waviness with a wavelength of $4 z_{3}$ as presented in Fig. 12. The rate of work done by oblique cutting is smaller than the rate of work done by wire tension as seen in equation (23). Mechanism 2 occurs when the wire tension is high, which leads to a stiff

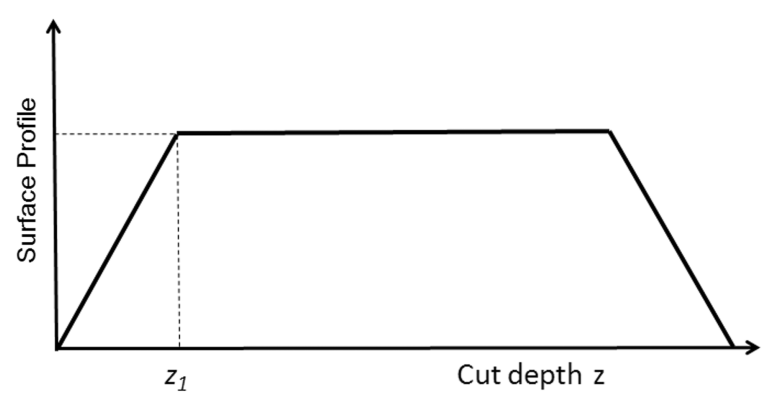

Fig. 10 Surface profile due to mechanism 1

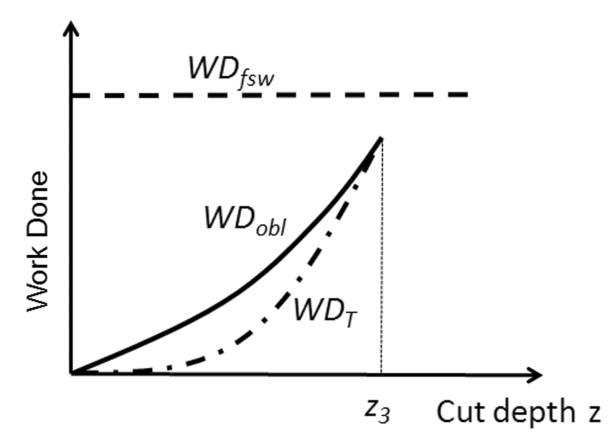

Fig. 11 Energy plot for mechanism 2

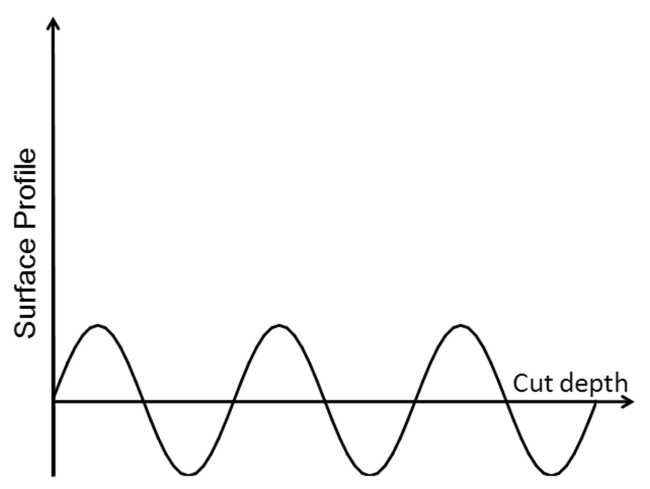

Fig. 12 Surface profile due to mechanism 2 
wire. The amplitude of the waviness obtained in mechanism 2 will be smaller than the amplitude of the wire drift in mechanism 1

$$
\begin{aligned}
& W D_{\text {obl }}=W D_{T} \\
& \frac{\partial W D_{\text {obl }}}{\partial z}<\frac{\partial W D_{T}}{\partial z}
\end{aligned}
$$

\section{RESULTS AND DISCUSSION}

The upper bound for the wire deflection in the cutting plane $X-Z$ and the oblique plane $X-Y$ was derived using the string model. A lateral deflection model, including oblique cutting of abrasive grits, energetic terms affecting wire deflection, and the mechanisms based on the energetic terms and governing the surface profile, was derived.

The profiles of the wire marks obtained from the cut surfaces in the $X-Z$ plane are presented in Fig. 13. The discrete data points are measurements obtained from the cut surfaces of each test, while the continuous curves are the string model predictions. The distributed load on the wire was obtained using equation (24). The wire bow angle $\alpha$ was measured from the images captured during the test. The distributed load $w$, wire tension $T$, cut length $L_{0}$, and span length $L$ were inserted in equation (8) to obtain the string model predictions for the wire bow, as presented in Fig. 13. The string model estimations for the wire profile in the $X-Z$ plane were satisfactory

$$
w=\frac{2 T \sin \alpha}{L_{0}}
$$

The doubling of tension decreased the wire bow amplitude almost by half, while increasing the feed speed increased the wire bow amplitude as seen in Fig. 13. These trends are captured by the string model very well, as also seen in Fig. 13.

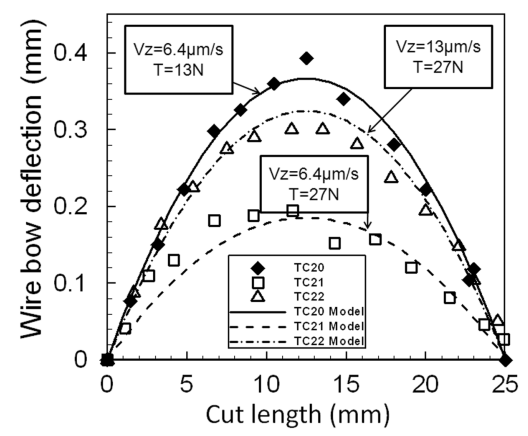

Fig. 13 Variation of wire bow as a function of wire tension and feed speed in the cut plane $(X-Z)$
The surface profiles obtained from profilometer measurements for each test are presented in Figs 14 to 16 . The proportionality factor $\beta$ in equations (11) and (12) was determined from the slopes of the dashed lines representing the out-of-plane deflection of the wire due to oblique forces, as presented in Figs 14 to 16 . The average of the slopes of these lines was attained as $\beta=0.0123$. The energy plots of the tests are presented in Figs 17 to 19, respectively. All of the energy plots showed mechanism 1 behaviour, in which the work done by oblique cutting is

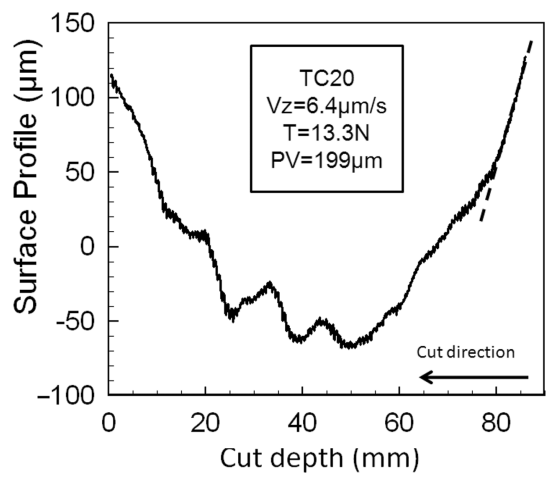

Fig. 14 Surface long waviness profile of test TC20

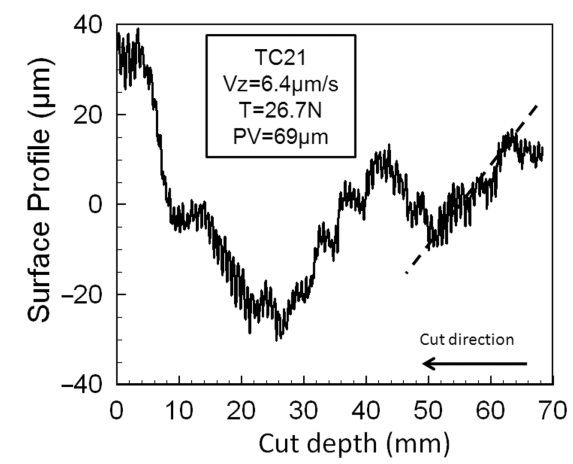

Fig. 15 Surface long waviness profile of test TC21

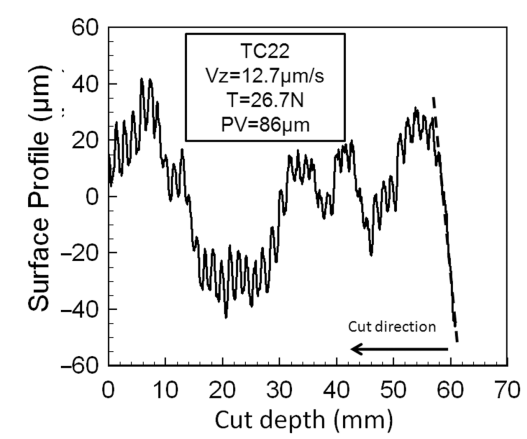

Fig. 16 Surface long waviness profile of test TC22 


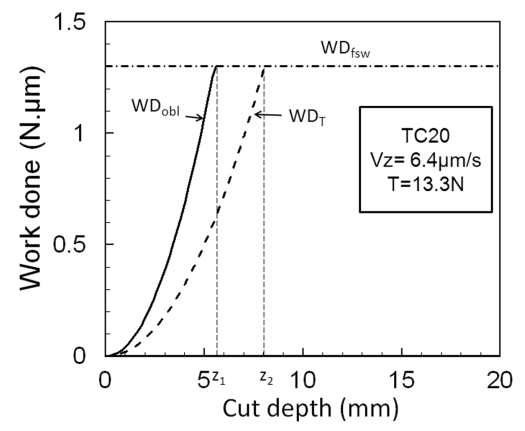

Fig. 17 Energy plot of test TC20

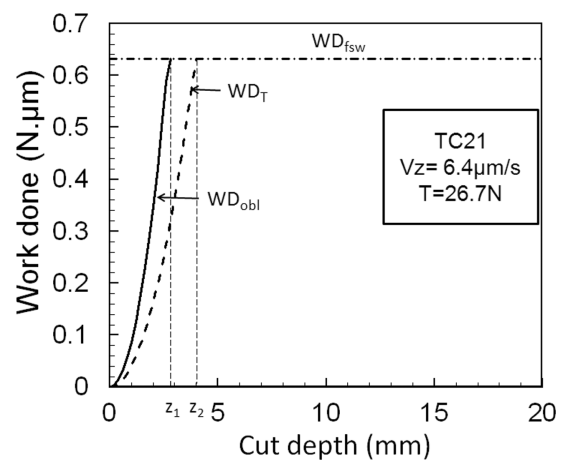

Fig. 18 Energy plot of test TC21

limited by the work done by the free-standing wire, as in Fig. 9. Although all of the energy plots showed mechanism 1 behaviour, increased wire tension or feed speed had a marked effect on the long waviness, which can still be explained by the waviness model. The increase of wire tension leads to a stiff wire, which will lead to mechanism 2, a wavy surface, while the increase of feed speed increases the oblique cutting forces, which will lead to mechanism 1 . All the tests in this work had mechanism 1 type energy plots as presented in Fig. 9. However, the increase of wire tension decreases the difference $z_{2}-z_{1}$, which drives the system towards mechanism 2 and, ultimately, when $z_{2}-z_{1}=0$, mechanism 2 will be obtained energetically, as in Fig. 11. There is not a strict boundary between mechanism 1 and mechanism 2 . While a system is energetically in mechanism 1 , because of high wire tension the difference $z_{2}-z_{1}$ will decrease, driving the system towards mechanism 2, as in Fig. 20 for TC21 with respect to TC20. Increase of feed speed will increase the oblique cutting forces, driving the system towards mechanism 1 and increasing the difference $z_{2}-z_{1}$, as seen in Fig. 20 for TC22 with respect to TC21.

Test TC20 was done with wire tension $T=13.3 \mathrm{~N}$, while TC21 was done with $T=26.7 \mathrm{~N}$; both tests had

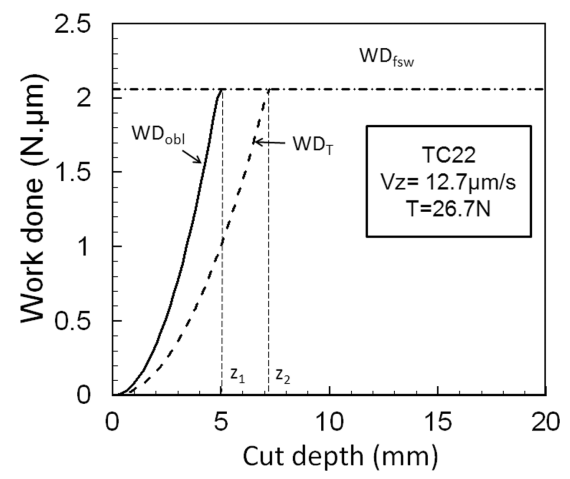

Fig. 19 Energy plot of test TC22

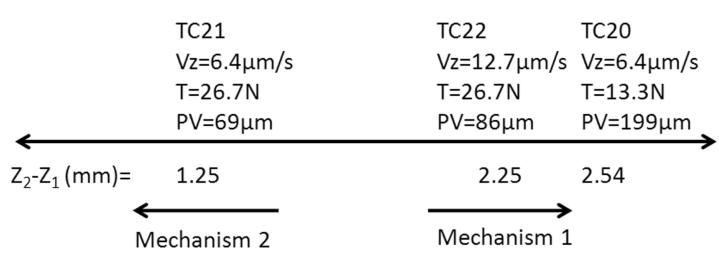

Fig. 20 Domains for long waviness mechanisms

the same wire speed $V_{x}$ and feed speed $V_{z}$. The increase of wire tension decreased the difference $z_{2}-z_{1}$ for TC21 with respect to TC20, driving test TC21 towards mechanism 2, as seen in Fig. 20. Test TC20, being closer to mechanism 1, had a step-like surface, as in Fig. 14, which complies with the expectations for a step-like surface for mechanism 1 as presented in Fig. 10. Test TC21, being closer to mechanism 2 with respect to TC20, had a wavy surface as presented in Fig. 15. The peak-to-valley (PV) value, which is the height difference between the highest and lowest points on a surface profile, is also affected by the change in wire tension. Experiment TC20, having a lower wire tension of $T=13.3 \mathrm{~N}$, had a higher $\mathrm{PV}=199 \mu \mathrm{m}$, while experiment TC21, having a higher wire tension of $T=26.7 \mathrm{~N}$, had a lower $\mathrm{PV}=69 \mu \mathrm{m}$. The increase of wire tension $T$ decreased the PV value, which is expected as mechanism 1 creates a high-amplitude step while mechanism 2 creates lower-amplitude waviness.

Test TC21 was done with a feed speed of $V_{z}=6.4 \mu \mathrm{m} / \mathrm{s}$, while TC22 was done with $V_{z}=12.7 \mu \mathrm{m} / \mathrm{s}$; both had the same wire speed and tension. The increase of feed speed increased the oblique cutting forces, driving TC22 towards mechanism 1 . The increase of feed speed increased the difference $z_{2}-z_{1}$ for TC22 with respect to TC21, driving TC22 towards mechanism 1, as seen in Fig. 20. The PV value was also affected by the change of feed speed. Experiment TC21, having a lower feed speed of 
$\mathrm{V}_{\mathrm{z}}=6.4 \mu \mathrm{m} / \mathrm{s}$, had a lower $\mathrm{PV}=69 \mu \mathrm{m}$, while experiment TC22, having a higher feed speed of $V_{z}=12.7 \mu \mathrm{m} / \mathrm{s}$, had a higher $\mathrm{PV}=86 \mu \mathrm{m}$. The increase of feed speed increased the PV value, which is expected as mechanism 1 creates a high-amplitude step while mechanism 2 creates lower-amplitude waviness.

Figure 20 shows that as the difference $z_{2}-z_{1}$ increased, the PV value increased for all three experiments and the system evolved towards mechanism 1 , while decreasing difference $z_{2}-z_{1}$ led to mechanism 2. High wire tension reduced the PV value, while high feed rate increased the PV value. The increase of PV value will lead to more post grinding and polishing of the cut surface and an increase in expenses. In order to increase the efficiency without increasing the PV value, the wire tension can be increased proportional to feed speed.

\section{CONCLUSIONS}

The long waviness on the cut surface induced by the wire saw process has been investigated in this study. Experimental work including different wire tensions and feed speeds was conducted. The string model was used to model the marks made by the bow of the wire on the cut surface and satisfactory results were obtained. The increase of tension decreased the wire bow amplitude, while increase of feed speed increased the wire bow amplitude, which was predicted by the string model and observed in the experiments.

The long waviness formation has been explained with energetic terms that define two mechanisms leading to different surface profiles. The defined mechanisms were used to explain the evolution of the surface profile and peak-to-valley value with respect to process parameters. The increase of wire tension led to a wavy surface with a lower peak-to valley-value, while decreasing wire tension led to a step-like surface with a high peak-to-valley value. The increase of feed speed led to a high peak-tovalley value. The increase of peak-to-valley value leads to more post grinding and polishing of the cut surface and increases in expenses. In order to increase the efficiency without increasing the peakto-valley value, the wire tension can be increased proportional to feed speed.

\section{ACKNOWLEDGEMENT}

This work was supported by the National Science Foundation (NSF) of the USA. This work is a part of the author's PhD study at Iowa State University, USA.
(C) Authors 2011

\section{REFERENCES}

1 Zhu, L. and Kao, I. Galerkin based modal analysis on the vibration of wire-slurry system in wafer slicing using a wire saw. J. Sound Vib., 2005, 283, 589-620.

2 Clark, W. I., Shih, A. J., Hardin, C. W., Lemaster, R. L., and McSpadden, S. B. Fixed abrasive diamond wire machining - Part II: experiment design and results. Int. J. Machine Tools Mf., 2003, 43, 533-542.

3 Ge, P. Q., Zhang, L., Gao, W., and Liu, Z. C. Development of endless diamond wiresaw and sawing experiments. Mater. Sci. Forum, 2004, 471472, 481-484.

4 Clark, W. I., Shih, A. J., Hardin, C. W., Lemaster, R. L., and McSpadden, S. B. Fixed abrasive diamond wire machining - Part I: process monitoring and wire tension force. Int. J. Machine Tools Mf., 2003, 43, 523-532.

5 Bhagavat, M., Prasad, V., and Kao, I. Elasto-hydrodynamic interaction in the free abrasive wafer slicing using a wiresaw: modeling and finite element analysis. Trans. ASME, Tribol. Div., 2000, 122, 394-404.

6 Hardin, C. W., Qu, J., and Shih, A. J. Fixed abrasive diamond wire saw slicing of single-crystal silicon carbide wafers. Mater. Mfg Process, 2004, 19, 355-367.

7 Moller, J. H. Basic mechanisms and models of multiwire sawing. Adv. Engng Mater., 2004, 6, 501-513.

8 Liu, B. C., Zhang, Z. P., and Sun, Y. H. Sawing trajectory and mechanism of diamond wire saw. Key Engng Mater., 2004, 259-260, 395-400.

9 Wei, S. and Kao, I. Analysis of stiffness control and vibration of wire in wiresaw manufacturing process. Proc. ASME, Mfg Sci. Engng Div., 1998, 8, 813-818.

10 Wei, S. and Kao, I. Vibration analysis of wire and frequency response in the modern wire saw manufacturing process. J. Sound Vib., 2000, 231, 1383-1395.

11 Meng, J. F., Li, J. F., Ge, P. Q., and Zhou, R. Research on endless wire saw cutting of $\mathrm{Al}_{2} \mathrm{O}_{3} / \mathrm{TiC}$ ceramics. Key Engng Mater., 2006, 315-316, 571-574.

12 Liu, W. J., Pei, Z. J., and Xin, X. J. Finite element analysis for grinding and lapping of wire-sawn silicon wafers. J. Mater. Process Technol., 2002, 129, 2-9.

13 Pei, Z. J., Xin, X. J., and Liu, W. Finite element analysis for grinding of wire-sawn silicon wafers: a designed experiment. Int. J. Machine Tools Mf., 2003, 43, 7-16.

14 Pei, Z. J., Kassir, S., Bhagavat, M., and Fisher, G. R. An experimental investigation into soft-pad grinding of wire-sawn silicon wafers. Int. J. Machine Tools Mf., 2004, 44, 299-306.

15 Xin, X. J., Pei, Z. J., and Liu, W. Finite element analysis on soft-pad grinding of wire-sawn silicon wafers. J. Electron. Packag., 2004, 126, 177-185.

16 Sun, X., Pei, Z. J., Xin, X. J., and Fouts, M. Waviness removal in grinding of wire-sawn silicon wafers: 3D finite element analysis with designed experiments. Int. J. Machine Tools Mf., 2004, 44, 11-19. 
17 Jiao, Y., Pei, Z. J., Lei, S., Lee, E. S., and Fisher, G. R. A fuzzy adaptive network model for waviness removal in grinding of wire-sawn silicon wafers. J. Mfg. Sci. Engng, Trans. ASME, 2006, 128, 938-943.

18 Bastawros, A. F., Chandra, A., and Shih, A. Analysis of surface waviness during wire saw cutting of ductile materials. In Proceedings of 2006 NSF Design Service and Manufacturing Grantees and Research Conference, St. Louis, Missouri, 24-27 July 2006, (CD).

19 Material Property Data. Alumina 99.9\%. http:// www.matweb.com/search/DataSheet.aspx?MatGU ID=c8c56ad547ae4cfabad15977bfb537f1 (accessed: 20/01/2008).

20 Cook, R. F. and Pharr, G. M. Direct observation and analysis of indentation cracking in glasses and ceramics. J. Am. Ceram. Soc., 1990, 73, 787-817.

\section{APPENDIX}

\section{Notation}

E Young's modulus

$H \quad$ hardness

$H_{\mathrm{s}} \quad$ height of the sample
$K_{\mathrm{IC}} \quad$ fracture toughness

$L \quad$ length of the wire

$L_{\mathrm{O}} \quad$ cut length of the sample

$R(z) \quad$ lateral reaction on the wire

$T \quad$ wire tension

$V_{x} \quad$ wire axial speed

$V_{z} \quad$ feed rate

$w \quad$ distributed load on the wire

$W D_{\text {fsw }}$ work done by free-standing wire

$W D_{\text {obl }}$ work done by oblique cutting forces

$W D_{\mathrm{T}} \quad$ work done by wire tension

$z \quad$ cut depth

$z_{1} \quad$ critical cut depth at which $W D_{\mathrm{fsw}}=W D_{\text {obl }}$

$\alpha \quad$ wire bow angle

$\beta \quad$ proportionality factor

$\delta(x) \quad$ string deflection

$\theta_{\mathrm{f}} \quad$ angle between the wire and the horizontal line in the $X-Y$ plane

$v \quad$ Poisson's ratio

$\sigma_{\text {fr }} \quad$ tensile strength 